\title{
IDENTIFICACIÓN DE FACTORES DE RIESGO PARA LA PRESENTACIÓN DE CETOSIS SUBCLÍNICA EN GANADO BOVINO LECHERO
}

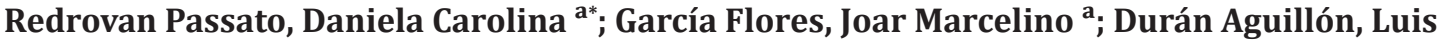 \\ Enrique $^{b}$ \\ ${ }^{a}$ Universidad de las Américas, Vía a Nayón, Quito , Ecuador. \\ b Universidad de las Fuerzas Armadas - Autopista General Rumiñahui S/N y Ambato, Sangolquí, Quito, \\ Ecuador.
}

Ingresado: 21/10/2019

\section{Resumen}

Un mal manejo sanitario, reproductivo y multifuncional en la ganadería lechera causa una mayor incidencia de problemas metabólicos como la cetosis clínica y subclínica, que son considerados como críticos durante el periodo de transición. La cetosis subclínica representa un desafío para las personas que la enfrentan en sus vacas porque está subdiagnosticada y genera gran impacto económico relacionado con trastornos de salud que reflejan pérdidas en eficiencia reproductiva, producción lechera y bienestar animal. El objetivo del estudio consistió en analizar la producción lechera, la condición corporal y número de partos como factores de riesgo para la presencia de cetosis subclínica en vacas lecheras y determinar su asociación en una explotación de leche. Mediante criterios de inclusión y exclusión, se seleccionó registros con un muestreo de orina de 29 vacas en el preparto, parto, y 15, 30, 60 y 90 días post parto. Esta información se analizó con la prueba estadística Chi cuadrado de Pearson cuyos resultados muestran que la presencia de cetosis subclínica depende de la producción lechera a los 60 días post parto $(\mathrm{p}<0,05)$ y a los 90 días post parto $(\mathrm{p}<0,05)$. El valor de 0,57 se obtuvo para la prueba $\mathrm{V}$ de Cramer a los 60 días post parto y un valor de 0,61 a los 90 días post parto, demostrando un 57\% - 61\% de asociación entre la producción lechera y la cetosis subclínica, considerándola como factor de riesgo. Se concluyó que la producción lechera tiene relación directa con la enfermedad, al mismo

\footnotetext{
* Correspondencia a: Universidad de las Américas, Vía a Nayón, Quito 170124, Ecuador. Teléfono: +593998426383.

Correo electrónico: dani_redrovan@hotmail.com
}

Aceptado: 29/04/2020

tiempo que se categorizó a las variables condición corporal y número de partos como independientes y sin asociación entre sí.

Palabras clave: Bovinos, cetosis, condición corporal, producción lechera.

\section{IDENTIFICATION OF RISK FACTORS FOR SUBCLINICAL KETOSIS IN DAIRY CATTLE}

\begin{abstract}
Poor sanitary, reproductive and multifunctional management in dairy cattle causes a higher incidence of metabolic problems such as clinical and subclinical ketosis during the transition period that is considered critical. Subclinical ketosis represents a challenge for people who face it in their cows, because it is underdiagnosed, generating economic impact related to health disorders that reflect losses in reproductive efficiency, dairy production and animal welfare. The aim of this study was to analyze milk production, body condition and number of births as risk factors to cause subclinical ketosis in dairy cows, establish their association at a milk farm.
\end{abstract}

By using inclusion and exclusion criteria, records were selected through urine sampling and recorded in prepartum, partum, and 15, 30, 60 and 90 days postpartum from which 29 cows were selected. This information was analyzed with Pearson's Chi square statistical test whose results showed that the presence of subclinical ketosis depends on milk production at 60 days postpartum $(\mathrm{p}<0,05)$ and at 90 days post-partum ( $\mathrm{p}<$ $0,05)$. The value of 0,57 was obtained for the V-test 
of Cramer at 60 days postpartum and a value of 0,61 at 90 days postpartum, demonstrating a $57 \%$ - $61 \%$ association between dairy production and subclinical ketosis, considering dairy production as a risk element. The study concluded that milk production is directly related to the disease, and the variables body condition and number of births were categorized as independent and unassociated.

Keywords: Cattle, ketosis, body condition, milk production.

\section{INTRODUCCIÓN}

Concentraciones anormalmente elevadas de cuerpos cetónicos, acetona, ácido acetoacético y ácido beta-hidroxibutírico se conoce como cetosis [1]. Se manifiesta con pérdida progresiva de apetito, disminución de la producción lechera, signos vitales anormales, depresión, motilidad ruminal disminuida y la muerte. Se clasifica como clínica y subclínica, dependiendo de los niveles en fluidos de todo el cuerpo y de acuerdo a la ausencia o presencia de signos clínicos [2].

Existen dos tipos de cetosis de acuerdo al origen de los cuerpos cetónicos; la primaria (tipo I) es resultado de una hipoglucemia alimenticia, mientras que en la secundaria (tipo II), el organismo no puede convertir el alimento en energía por problemas asimilativos, digestivos o de consumo [3]. Ambos tipos de cetosis se presentan con un mismo cuadro sintomático clínico o subclínico dificultando el tratamiento, ya que no se conoce bien la causa [4]. La cetosis puede ser considerada secundaria a cualquier enfermedad que origine ayunos prolongados o disminución del consumo de alimento durante el periodo de transición [5].

El periodo entre preparto y lactación temprana (periodo de transición) es un momento crítico, en el que se observa la mayor incidencia de problemas metabólicos como la cetosis clínica y subclínica [6]. El manejo sanitario, reproductivo y multifuncional en la ganadería durante este periodo es importante, especialmente en vacas de alta producción (> 29 litros), ya que estas suelen tener una forma anormal de regular la producción de cuerpos cetónicos [7]. Las pérdidas económicas que genera la enfermedad se relacionan con inadecuados manejos que predisponen trastornos de salud reflejando disminución en eficiencia reproductiva, producción lechera y bienestar animal $[8,9]$.

La estrecha relación entre la cetosis (clínica o subclínica) y la gestación avanzada, parto, puerperio, producción lechera, manejo, alimentación y fertilidad constituye un desafío para las personas que la enfrentan en sus vacas, por los gastos económicos que implica [4]. El problema radica en que, al no detectar una cetosis subclínica, los inconvenientes reproductivos y una baja producción lechera se convierten en una dificultad para el médico veterinario y el ganadero porque ignoran el origen metabólico, se confunde el diagnóstico y aumentan los gastos tratando de solucionar el problema.

El diagnóstico temprano de la enfermedad mediante signos clínicos no es tan efectivo ya que son poco específicos, llevando a una confusión con otras patologías como la hipocalcemia e hipomagnesemia [10]. El diagnóstico se realiza empleando varios métodos de medición de cuerpos cetónicos en sangre, orina y leche. Para esto, se conoce que en bovinos, los valores normales de cuerpos cetónicos en orina son: $0 \mathrm{mmol} / \mathrm{L}-0,17$ $\mathrm{mmol} / \mathrm{L}$ de acetona, $0 \mathrm{mmol} / \mathrm{L}-0,35 \mathrm{mmol} / \mathrm{L}$ de ácido acetoacético y $0 \mathrm{mmol} / \mathrm{L}-1,18 \mathrm{mmol} / \mathrm{L}$ de ácido Beta-hidroxibutírico, los cuales se usaron de referencia para el presente estudio. Cuando estos aumentan se habla de cetosis subclínica, ya que para llegar a una cetosis clínica se necesitan $22 \mathrm{mmol} / \mathrm{L}$ de ácido acetoacético y la presencia de signos clínicos [2].

Las tiras reactivas comerciales son tests de orina rápidos y fáciles de utilizar para la determinación de 10 diferentes parámetros en humanos, dentro de los cuales está el cuerpo cetónico, ácido acetoacético, diferente al que generalmente se mide en tiras reactivas hechas para bovinos, en las cuales se mide el Beta-hidroxibutírico. Provee información diagnóstica para anormalidades metabólicas, enfermedades o desórdenes en riñones, hígado, tracto urinario, problemas hemolíticos y en el equilibrio ácido-base [11]. Se utiliza el principio del reactivo rothera que aplicado en orina tiene una sensibilidad del $49 \%$ $76 \%$ y una especificidad del 96\% - 99\% [12].

Obtener información sobre factores de riesgo para la presentación de cetosis es relevante y determinante, porque la patología afecta la eficiencia de los rebaños lecheros [13]. Al conocer los factores de riesgo de la enfermedad, se puede hacer un mejor monitoreo, manejar procedimientos de prevención y reducir el impacto económico que genera la enfermedad. La cetosis subclínica tiene un impacto de carácter económico por pérdidas directas e indirectas. 
Aunque el Ecuador es un país agropecuario, no cuenta con trabajos que profundicen en problemas de origen metabólico, por lo que el presente estudio puede ayudar en predios ganaderos que presenten problemas o que quieran aportar en futuras investigaciones. El objetivo del presente trabajo fue analizar la producción lechera, la condición corporal y número de partos como factores de riesgo para la presencia de cetosis subclínica en vacas lecheras y determinar la asociación entre las variables.

\section{METODOLOGÍA}

El presente estudio fue de tipo observacional ambispectivo, que se llevó a cabo en una explotación lechera en la parroquia Alóag del cantón Mejía. Se tomaron los registros de 29 vacas en preparto hasta los 90 días de producción, con 6 muestras de orina y 105 días de estudio por vaca. Para el diagnóstico, se utilizaron tiras reactivas de orina Combina 10 M HUMAN, que miden cuerpos cetónicos (ácido acetoacético) y se leen mediante una escala colorimétrica. La explotación lechera contaba con un aproximado de 100 animales de los cuales destacan grupos específicos como: 60 adultos, 48 (45-55) vacas en producción, 11 vacas en seco, 12 vaconas y 10 vientres inseminadas o por inseminar.

De esta población, se seleccionó la muestra y se tomaron en cuenta criterios de inclusión y exclusión sobre los registros aplicados a los animales que fueron escogidos para el monitoreo. Se manejaron 18 registros de los cuales 9 fueron tomados en cuenta para el estudio: registro de evaluación de cetosis subclínica en preparto, parto, y 15, 30, 60 y 90 días post parto; registros de: litros de leche semanales, de nutrición, y de vacas y vientres que entran al periodo de seco con su fecha estimada de partos y de su último chequeo ginecológico.

Todo el muestreo se realizó bajo un esquema de tiempo, en el que cada vaca en seco era candidata para entrar en el estudio. El predio contó con protocolos para medir la condición corporal y obtener la muestra de orina para el diagnóstico de cetosis subclínica con tiras reactivas en la primera toma de muestra. Para la segunda toma de muestra se adicionaron parámetros del parto y finalmente, de la tercera hasta la sexta toma de muestra se agregaron registros de la producción lechera.

\section{Toma de muestra}

El masaje en el periné es la forma más común de toma de muestra de orina. Para este método se necesita un procedimiento de contención como una manga de manejo y se recomienda meter varios animales en la manga para que las vacas se sientan tranquilas [14]. Primero se limpió el área de la vulva que suele encontrarse con heces; con guantes de manejo se procedió a masajear enérgicamente el área que se encuentra $5 \mathrm{~cm}$ por debajo de la vulva (periné) hasta estimular lo suficiente para que el animal orine y recolectar la orina en vasos recolectores estériles para llevarla inmediatamente a un lugar cerrado.

Cronológicamente, la primera muestra se tomó 15 días antes del parto al momento de dar el balanceado en los collarines del ordeño; la segunda muestra se tomó el día del parto hasta máximo el tercer día post parto después del ordeño del calostro. Las siguientes muestras, tercera (15 días post parto), cuarta (30 días post parto), quinta (60 días post parto) y sexta (90 días post parto) se tomaron después del segundo ordeño del día antes de que salgan del collarín.

\section{Análisis de las muestras con tiras reactivas}

El análisis se llevó a cabo con las muestras frescas de orina en el momento en que se las obtuvo. En un área limpia, se utilizó una tira reactiva que se sumergió de 1 a 2 segundos en la muestra de orina, lo suficiente para que se mojen todas las almohadillas de la misma. Se escurrió la orina restante de la tira para colocarla horizontalmente sobre una superficie plana y evitar que los reactivos se mezclen entre almohadillas; los resultados se leyeron 1 minuto después.

El test se basa en reacciones bioquímicas y químicas que producen cambios de color en cada una de las almohadillas. Coloraciones que solo aparecieron en el borde de las almohadillas no se las consideró importantes y cualquier desviación de los colores normales fueron útiles para profundizar en el diagnóstico y posibles tratamientos [11]. Tomando en cuenta la última aclaración, se compararon las zonas reactivas con la escala de colores del envase y se anotaron los valores de cada uno de los 10 parámetros que mide la tira de acuerdo al tiempo del muestreo. Las tiras pueden detectar 4 grados de ácido acetoacético: 0,5 $\mathrm{mmol} / \mathrm{L}, 1,5 \mathrm{mmol} / \mathrm{L}, 5$ $\mathrm{mmol} / \mathrm{L} \mathrm{y} 15 \mathrm{mmol} / \mathrm{L}$. 


\section{Medición de condición corporal y registro de producción lechera}

Se utilizó el método de Edmonson [15] desarrollado en 1989, que es una adaptación de los sistemas neozelandeses para una evaluación visual. Se empleó la escala de 1 a 5 con ajustes de 0,25 puntos, donde 1 correspondía a una vaca esquelética o emaciada y 5 a una vaca obesa [16]. Se evaluó y registró la condición corporal de cada animal con cada muestreo de orina. Una persona fue encargada de realizar las mediciones, para evitar posibles variaciones en las mismas. Por otra parte, una vez comenzada la lactación, se registró el promedio semanal de litros de leche hasta cumplir los 90 días de producción.

\section{Análisis de registros y tabulación de datos}

Se realizó un levantamiento de información mediante la revisión de los registros seleccionados por criterios de inclusión. Todos los datos netos obtenidos fueron tabulados y ordenados para tener una secuencia lógica. Posteriormente, se analizaron los datos mediante razonamiento y asociación de eventos que coinciden en un mismo momento. Se utilizaron métodos estadísticos como el Chi Cuadrado de Pearson y V de Cramer para identificar la asociación de las variables y distinguir el factor de riesgo más predisponente para la presentación de cetosis subclínica.

\section{Análisis estadísticos}

Para llevar a cabo este estudio, se tomó en cuenta las siguientes variables: condición corporal, número de partos, producción lechera y la presencia de cetosis subclínica; cada una con sus respectivos tipos de variable, características, indicadores, unidades de medida y definiciones.

Para los resultados bivariados, se agrupó los datos colectados de acuerdo a su etapa fisiológica y apuntes prácticos del libro "El periparto de la vaca" [17] para aplicar pruebas estadísticas de nominales. Las agrupaciones se realizaron de acuerdo al número de partos; los animales del primer y segundo parto formaron el grupo $1 \mathrm{y}$ los animales del tercer, cuarto, quinto y sexto parto formaron el grupo 2 (Tablas 1, 2 y 3 ).

Tabla 1: Agrupación de cetosis subclínica.

\begin{tabular}{llll|}
$\begin{array}{l}\text { Cetosis Subclínica } \\
\text { (ácido acetoacético) }\end{array}$ & Grado & Sigla \\
\begin{tabular}{|lll}
$0 \mathrm{mmol} / \mathrm{L}=$ negativo & - & $\mathrm{N}$ \\
$0,5 \mathrm{mmol} / \mathrm{L}=$ positivo & Bajo & $\mathrm{PB}$ \\
$1,5 \mathrm{mmol} / \mathrm{L}=$ positivo & Medio & $\mathrm{PM}$ \\
$5 \mathrm{mmol} / \mathrm{L}=$ positivo & Medio - Alto & $\mathrm{PMA}$ \\
$15 \mathrm{mmol} / \mathrm{L}=$ positivo & Alto & $\mathrm{PA}$ \\
\hline
\end{tabular}
\end{tabular}

Nota: : Negativo a cetosis subclínica (N), positivo a cetosis subclínica en grado bajo (PB), positivo a cetosis subclínica en grado medio (PM), positivo a cetosis subclínica en grado medio-alto (PMA) y positivo a cetosis subclínica en grado alto (PA).

Tabla 2: Agrupación de condición corporal.

\begin{tabular}{|c|c|c|c|c|c|c|}
\hline Grado de CC & Preparto & Parto & $\begin{array}{c}15 \text { días post } \\
\text { parto }\end{array}$ & $\begin{array}{l}30 \text { días post } \\
\text { parto }\end{array}$ & $\begin{array}{c}60 \text { días post } \\
\text { parto }\end{array}$ & $\begin{array}{c}90 \text { días post } \\
\text { parto }\end{array}$ \\
\hline $\begin{array}{l}\text { Animales } \\
\text { delgados }\end{array}$ & $\begin{array}{l}\text { hasta } 3 \\
\text { puntos }\end{array}$ & $\begin{array}{l}\text { hasta } 3 \\
\text { puntos }\end{array}$ & $\begin{array}{l}\text { hasta } 3 \\
\text { puntos }\end{array}$ & $\begin{array}{l}\text { hasta } 3 \\
\text { puntos }\end{array}$ & $\begin{array}{l}\text { hasta } 3 \\
\text { puntos }\end{array}$ & $\begin{array}{l}\text { hasta } 3 \\
\text { puntos }\end{array}$ \\
\hline $\begin{array}{l}\text { Animales } \\
\text { óptimos }\end{array}$ & $\begin{array}{c}3,25-3,75 \\
\text { puntos }\end{array}$ & $\begin{array}{c}3,25-3,75 \\
\text { puntos }\end{array}$ & $\begin{array}{c}3,25-3,75 \\
\text { puntos }\end{array}$ & $\begin{array}{c}3,25-3,75 \\
\text { puntos }\end{array}$ & $\begin{array}{c}3,25-3,75 \\
\text { puntos }\end{array}$ & $\begin{array}{c}3,25-3,75 \\
\text { puntos }\end{array}$ \\
\hline $\begin{array}{l}\text { Animales } \\
\text { gordos }\end{array}$ & $\begin{array}{c}4 \text { puntos en } \\
\text { adelante }\end{array}$ & $\begin{array}{c}4 \text { puntos en } \\
\text { adelante }\end{array}$ & $\begin{array}{l}4 \text { puntos en } \\
\text { adelante }\end{array}$ & $\begin{array}{c}4 \text { puntos en } \\
\text { adelante }\end{array}$ & $\begin{array}{l}4 \text { puntos en } \\
\text { adelante }\end{array}$ & $\begin{array}{c}4 \text { puntos en } \\
\text { adelante }\end{array}$ \\
\hline
\end{tabular}

Fuente: Fernández M, Liz M, Hernández M [17]. 
Tabla 3: Agrupación de producción lechera.

\begin{tabular}{|c|c|c|c|c|c|}
\hline Grupo & $\begin{array}{l}\text { Nivel de } \\
\text { producción }\end{array}$ & $\begin{array}{l}\text { Litros de producción } 15 \\
\text { días post parto }\end{array}$ & $\begin{array}{l}\text { Litros de producción } 30 \\
\text { días post parto }\end{array}$ & $\begin{array}{l}\text { Litros de producción } 60 \\
\text { días post parto }\end{array}$ & $\begin{array}{l}\text { Litros de producción } 90 \\
\text { días post parto }\end{array}$ \\
\hline 1 & Baja & $\begin{array}{l}126 \text { L-266 L* } \\
(8-17) \mathrm{L} / \text { día }\end{array}$ & $\begin{array}{c}266 \text { L- } 350 \text { L* } \\
(18-23) \mathrm{L} / \text { día }\end{array}$ & $\begin{array}{l}343 \text { L-616 L* } \\
(11-20) \mathrm{L} / \text { día }\end{array}$ & $\begin{array}{l}357 \text { L- } 560 \text { L* } \\
(11-18) \mathrm{L} / \text { día }\end{array}$ \\
\hline 2 & Media & $\begin{array}{l}280 \mathrm{~L}-420 \mathrm{~L}^{*} \\
(18-28) \mathrm{L} / \text { día }\end{array}$ & $\begin{array}{c}364 \text { L-448 L* } \\
(24-29) \mathrm{L} / \text { día }\end{array}$ & $\begin{array}{l}644 \text { L-840 L* } \\
(21-28) \text { L/día }\end{array}$ & $\begin{array}{c}588 \text { L- } 845 L^{*} \\
(19-28) \mathrm{L} / \text { día }\end{array}$ \\
\hline 3 & Alta & $\begin{array}{l}\text { 434 L-532 L* } \\
\text { (29-35) L/día }\end{array}$ & $\begin{array}{l}449 \text { L-532 L* } \\
(30-35) \mathrm{L} / \text { día }\end{array}$ & $\begin{array}{l}841 \text { L- 1064 L* } \\
(29-35) \mathrm{L} / \text { día }\end{array}$ & $\begin{array}{l}846 \text { L- } 1036 \text { L* }^{*} \\
(29-35) \mathrm{L} / \text { día }\end{array}$ \\
\hline
\end{tabular}

Fuente: Fernández M, Liz M, Hernández M [17].

Nota: Para formar los rangos de litros acumulados, se tomó en cuenta los litros diarios de cada animal en los primeros 15 días y se los sumó. Para los 30 días, se acumularon los 15 días siguientes; para los 60 días, se sumaron los 30 días siguientes y de la misma forma hasta llegar a los 90 días de producción.

Para la asociación de variables se utilizó tablas de contingencia y Chi cuadrado de Pearson, que miden si las variables son independientes entre sí; mientras que, se utilizó la prueba $V$ de Cramer que describe y evalúa los grados de asociación entre 2 variables, donde 0 indica nada de asociación y 1 indica totalmente asociado [18].

\section{RESULTADOS Y DISCUSIÓN}

La cetosis subclínica en comparación al número de partos y condición corporal al preparto, parto, y 15, 30, 60 y 90 días post parto demostró independencia entre las variables ( $p>0,05)$. Su asociación se midió con la prueba V de Cramer, cuyos resultados fueron más cercanos al cero, demostrando que no hay asociación entre la cetosis subclínica, el número de partos y la condición corporal en el mismo periodo de tiempo. Es por ello que no se los consideró como factores de riesgo predisponentes para la presencia de cetosis subclínica.

Se presume que el tamaño muestral tiene gran influencia en estos resultados, ya que estudios con un mayor tamaño de muestra presentan resultados diferentes. Una investigación con 1.715 animales realizada en Holanda por Vanholder, Papen, Bemers, Vertenten, y Berge [19], explica que vacas de segundo parto tienen más probabilidad de presentar cetosis subclínica, a diferencia de animales de tercer parto en adelante que tienen más probabilidad de presentar una cetosis clínica.

El mismo estudio menciona que vacas en preparto con una condición corporal moderada de 3,25 a 3,75 puntos o gordas de 4 puntos en adelante tienen más probabilidad de desarrollar cetosis subclínica que vacas delgadas con condición corporal igual o menor a 3 puntos, debido a la movilización de grandes cantidades grasas [19]. Por otra parte, tanto animales delgados como animales gordos, tienden a presentar cetosis y otros problemas metabólicos [7].

Para tener un buen inicio de lactación, la naturaleza del bovino considera necesario mantener concentraciones elevadas de grasa en el calostro y leche de lactación temprana, y así cumplir con los requerimientos energéticos del ternero [20]. Para que esto suceda, el cuerpo debe tener valores altos de ácidos grasos no esterificados (AGNE), por lo que la movilización lipídica es elevada causando cetosis y otros trastornos metabólicos al inicio de la producción. La oxidación de AGNE causa depresión de ingesta del alimento en las últimas 4 semanas de gestación [21].

En este caso, la cetosis subclínica y la producción lechera demostraron independencia entre las variables a los 15 y 30 días post parto $(p>0,05)$. De igual manera, el resultado de asociación fue cercano al cero, por lo que no se los consideró como factores de riesgo predisponentes para la presencia de cetosis subclínica. Sin embargo, otros estudios mencionan que al momento del parto y en los primeros días de lactación, existe una probabilidad elevada de presentar cetosis subclínica por la producción de calostro atribuyéndose a un 6,7\% de grasa [19].

En cuanto al Chi Cuadrado de Pearson entre la cetosis subclínica y la producción lechera se demostró que las variables son dependientes entre sí, con un resultado de $\mathrm{p}=0,02$ a los 60 días post parto y $\mathrm{p}=0,014$ a los 90 días post parto. Usualmente se reporta un $5 \%$ de cetosis subclínica, pero al monitorear las primeras 3 semanas de producción, seguramente el valor incrementaría hasta un $40 \%$ [22]. 


\section{0 días post parto}

Oetzel [9] recomienda vigilar la enfermedad hasta los 50 días post parto; sin embargo, se logró distinguir que al continuar el monitoreo, todavía se observaba la enfermedad. En la Tabla 4 se puede apreciar que el $100 \%$ de los animales de producción baja (343 L - 616 L) y el $84,6 \%$ de los animales de producción media (644 L - $840 \mathrm{~L}$ ) son positivos a cetosis subclínica, a diferencia de los animales de producción alta (841L - $1.064 \mathrm{~L})$ donde solo el $30 \%$ fue positivo. Un valor de 0,57 se obtuvo para la prueba $\mathrm{V}$ de Cramer demostrando un 57\% de asociación entre la cetosis subclínica y la producción lechera hasta los 840 litros, es decir, animales que tendrán una lactación aproximada de 5.088 L en 305 días de producción.

Tabla 4: Asociación entre cetosis y producción lechera a los 60 días post parto.

\begin{tabular}{lccccc}
\hline & & $\mathbf{3 4 3}-$ & $\mathbf{6 4 4}-$ & $\mathbf{8 4 1}-$ & Total \\
& & $\mathbf{6 1 6} \mathbf{L}$ & $\mathbf{8 4 0} \mathbf{L}$ & $\mathbf{1 . 0 6 4} \mathbf{~}$ & \\
\hline $\begin{array}{l}\text { Cetosis } \\
\text { 60 días }\end{array}$ & Positivo & $100 \%$ & $84,6 \%$ & $30 \%$ & 15 \\
postparto & Negativo & $0 \%$ & $15,4 \%$ & $70 \%$ & 9 \\
\hline Total & & $100 \%$ & $100 \%$ & $100 \%$ & 24
\end{tabular}

\section{0 días post parto}

Saborio y Sánchez [23] mencionan que, vacas con mayor producción lechera tienden a presentar cetosis, a diferencia de vacas de menor producción. En la Tabla 5 se observa que el $90 \%$ de los animales con una producción media (588 L - 845 L) fueron positivos a cetosis subclínica, a diferencia de lo que se indica en otros estudios. Por otra parte, el $100 \%$ de los animales con producción baja $(357 \mathrm{~L}-560 \mathrm{~L})$ y el $66,7 \%$ de los animales con producción alta (846 L - 1.036 L) presentaron un resultado negativo a cetosis subclínica. Un valor de 0,61 se obtuvo para la prueba V de Cramer, demostrando un $61 \%$ de asociación entre la cetosis subclínica y la producción lechera de 588 hasta 845 litros, es decir, animales que tendrán una lactación aproximada de 5.118 L en 305 días de producción.

Tabla 5: Asociación entre cetosis y producción lechera 90 días post parto.

\begin{tabular}{lccccc}
\hline & & $\mathbf{3 5 7}-$ & $\mathbf{5 8 8}-$ & $\mathbf{8 4 6}-$ & Total \\
\hline $\begin{array}{l}\text { Cetosis } \\
\text { 90 días }\end{array}$ & Positivo & $0 \%$ & $90 \%$ & $33,3 \%$ & 13 \\
postparto & Negativo & $100 \%$ & $10 \%$ & $66,7 \%$ & 10 \\
\hline Total & & $100 \%$ & $100 \%$ & $100 \%$ & 23
\end{tabular}

Con base en los resultados obtenidos en este estudio, se considera a la producción lechera a los 60 y 90 días post parto como factor de riesgo predisponente para la presencia de cetosis subclínica. La mayor parte de casos de cetosis subclínica se manifiestan a las dos semanas post parto, y se encuentran asociados a vacas de alta productividad y a una deficiencia en su alimentación [10], ya que el tiempo posiblemente coincide con el pico de producción o con el nadir (máximo valor negativo) del balance energético negativo (BEN).

En el presente estudio se obtuvieron resultados diferentes a los trabajos de investigación de autores como Saborio y Sánchez [23], Duffield [10] y Oetzel [9], lo que puede ser debido a una nutrición post parto (70\% mezcla forrajera y 30\% suplementos) que se le suministra a toda vaca después del parto, en la explotación lechera. Se inicia con la toma de propilenglicol por ordeño durante tres días, balanceado regular que puede ir de $0 \mathrm{~kg}$ hasta $6 \mathrm{~kg}$ de ración diaria dependiendo de la producción de la vaca, y $200 \mathrm{~g}$ de grasa by-pass. Este protocolo se mantiene hasta los 100 días de producción lechera aproximadamente. Al encontrar cetosis subclínica pasado los 50 días de producción y en grupos de vacas donde normalmente no aparece (vacas de producción baja y media), es posible que no se suministre correctamente la ración alimenticia de acuerdo a la producción de los animales, y pueden estar recibiendo menos de lo que necesitan, reflejando un mal manejo de la nutrición en dichos grupos de vacas. Con el monitoreo permanente de cetosis se podría corregir este manejo inadecuado, evitando desordenes metabólicos subclínicos, que seguramente se verán reflejados en su producción total, reproducción y probabilidad de descarte.

\section{CONCLUSIONES}

Existe entre un 57\% - 61\% de asociación y dependencia entre la producción lechera y la cetosis subclínica. Se concluye que la producción lechera en rango medio, con una lactación proyectada de 5.088 L - 5.118 L en 305 días de producción es un factor de riesgo para la presentación de cetosis subclínica a los 60 y 90 días post parto. La condición corporal y el número de partos frente a la presencia de cetosis subclínica son variables independientes entre sí, y no se logró determinar asociación estadística entre las mismas en ninguno de los periodos estudiados.

El tiempo de muestreo fue un inconveniente, ya que para alcanzar un número grande de animales se necesitan años de estudio o varios predios 
con gran cantidad de animales e igual manejo. Consecuentemente, hubo métodos de estadística que fueron complicados de aplicar porque requieren de un tamaño de muestra más grande que los datos de los 29 animales que entraron en el estudio, es por esto que se decidió agrupar datos.

Finalmente, después de haber analizado tres factores de riesgo para la presencia de cetosis subclínica, se afirma que solo la producción lechera tiene relación directa con la enfermedad; indicando que las tiras reactivas combina 10 HUMAN pueden identificar cetosis subclínica en bovinos.

\section{REFERENCIAS}

[1] Rovers M. La cetosis en vacas lechera y el rol de la colina. ORFFA. [Internet]. 2014. Disponible en: https://orffa.com/es/la-cetosis-en-vacaslecheras-y-el-rol-de-la-colina/.

[2] Smith B. Trastornos de los sistemas orgánicos. En: Aleman M, Bannasch D, Barrington GM,et al, editores. Medicina Interna de Grandes Animales. 4ta. ed. España: Elsevier; 2010. p. 1339-1388.

[3] Perna R. Manejo Clínico del Sindrome Vaca Caída. 2da. ed. Buenos Aires: Inter-médica; 2009.

[4] Rosenberg G. Enfermedad con la participación de varios sistemas orgánicos. En: Dirksen G, Grunder HD, Stober M. Medicina interna y cirugía del bovino. 4ta. ed. Argentina: Inter-médica; 2005. p. 1190-1235.

[5] Noro M., Strieder C. Ketosis in Dairy Herds: Presentation and Control. J Dairy Vet Anim Res. 2012;7(6):299-302

[6] Du X., Chen L., Huang D., Peng Z., Zhao C., Zhang Y., Liu G. Elevated Apoptosis in the Liver of Dairy Cows with Ketosis. Cell. Physiol. Biochem. 2017; 43(2):568-578.

[7] Khalphallah A., Aamer A., Abdelall T., Elmeligy E., Oikawa S., Nakada K. Changes in clinical and blood lipid metabolism parameters in holstein dairy cattle during the transition period. Bulg. J. Vet. Med. 2017; 21(4): 420-428.

[8] Diaz M., Tovar I. Efecto de la cetosis subclinica posparto en la eficiencia reproductiva en vacas holstein fresian de la comarca lagunera. Revista Chapingo Serie Zonas Áridas. 2005; 1(4):41-46.

[9] Oetzel GR. Understanding the Impact of Subclinical Ketosis. Department of Medical Sciences, University of Wisconsin. [Internet]. 2015. Disponible en: https://pdfs.semanticscholar.org/ fd48/9fe9b96e0e6b5e029423596a79b6ecf81768. pdf?_ga $=2.4801075 .1461087073 .1573703027$ 1946002813.1573703027

[10] Duffield T., Lissemore K., McBride B., Leslie KE. Impact of hyperketonemia in early lactation dairy cows on health and production. J Dairy Sci. 2009; 92(2):571-580.

[11] HUMAN WD . Combina 10 M HUMAN. Magdeburgo (DEU): Aufbau Verlag; 2012. p. 1-10.

[12] Cucunubo L., Strieder-Barboza C., Wittwer F., Noro M. Diagnóstico de cetosis subclínica y balance energético negativo en vacas lecheras mediante el uso de muestras de sangre, orina y leche. Revista científica de la Facultad de Ciencias Veterinarias de la Universidad de Zulia. 2013; 23(2):111 - 119.

[13] Sepúlveda P., Wittwer F. ,Meléndez P. Período de transición. En:Importancia en la salud y bienestar de vacas lecheras. UAC [Internet]. 2017. Disponible en: https://www.consorciolechero.cl/ industria-lactea/wp-content/uploads/2017/11/ periodo-de-transicion.pdf

[14] Rocha E., Garzón J. Toma de muestra de orina en bovinos (masaje vulvar para inducir micción en vacas. Universidad Nacional de Colombia. [Internet]. 2013. Disponible en: http:// medicinaveterinariaydezootecnia.bogota.unal. edu.co/fileadmin/FVMZ/Servicios/bioetica/ Pro_autorizados/004_Toma_muestra_orina_bovinos. PDF[15] Edmonson A., Lean I., Weaver l., Farver T., Webster G. A body condition scoring chart for Holstein Dairy cows. J Dairy Sci. 1989; 72 (1): 6878.

[16] López F.J. Relación entre condición corporal y eficiencia reproductiva en vacas holstein. Dialnet. [Internet]. 2006. Disponible en: file:///C:/Users/ USUARIO/Downloads/Dialnet-RelacionEntreCond icionCorporalYEficienciaReproduct-6117891.pdf [17] Fernández M., Liz M., Hernández M. El periparto de la vaca: apuntes prácticos. 2 da. ed. Buenos Aires: SERVET; 2013.

[18] Rodríguez M.J. Mora R. En: Estadística informática : casos y ejemplos con el SPSS. Alicante : Publicaciones de la Universidad de Alicante. 2001. ISBN 84-7908-638-6, p. 3-17.

[19] Vanholder T., Papen J., Bemers R., Vertenten G., Berge A. Risk factors for subclinical and clinical ketosis and association with production parameters in dairy cows in the Netherlands. J Dairy Sci. 2015; 98(2): 880-888.

[20] Grigera J., Bargo F. Evaluación del estado corporal en vacas lecheras. Consultores Elanco Animal Health. [Internet]. 2005. Disponible en: www.produccion-animal.com.ar

[21] Allen M., Piantoni P. Metabolic control of feed intake: implications for metabolic disease of fresh cows. Vet Clin North Am Food Anim Pract. 2013; 29(2): 279-97.

[22] Leblanc S. Monitoring metabolic health of dairy cattle in the transition period. J Reprod Dev. 2010;56 Suppl: S29-35.

[23] Saborio A., Sánchez J. Prevalencia y factores 
de riesgo relacionados con la cetosis clínica y subclínica tipo I y II en un hato de vacas Jersey en Costa Rica. Agronomía Costarricense. 2013; 37(2): 17-29. 